\title{
Landmark-based Homologous Multi-point Warping Approach to 3D Facial Recognition Using Multiple Datasets
}

\author{
Olalekan Agbolade ${ }^{\text {Corresp., } 1}$, Azree Nazri ${ }^{\text {Corresp., } 1}{ }^{\text {, Razali Yaakob }}{ }^{1}$, Abdul Azim Abd Ghani ${ }^{2}$, Yoke Kqueen Cheah ${ }^{3}$ \\ 1 Department of Computer Science, Faculty of Computer Science \& IT, Universiti Putra Malaysia, Serdang, Selangor, Malaysia \\ 2 Department of Software Engineering, Faculty of Computer Science \& IT, Universiti Putra Malaysia, Serdang, Selangor, Malaysia \\ 3 Department of Biomedical Science, Faculty of Medicine and Health Sciences, Universiti Putra Malaysia, Serdang, Selangor, Malaysia \\ Corresponding Authors: Olalekan Agbolade, Azree Nazri \\ Email address: gs48997@student.upm.edu.my, azree@upm.edu.my
}

Over the years, neuroscientists and psychophysicists have been asking whether data acquisition for facial analysis should be performed holistically or with local feature analysis. This has led to various advanced methods of face recognition being proposed, and especially techniques using facial landmarks. The current facial landmark methods in 3D involve a mathematically complex and time-consuming workflow involving semi-landmark sliding tasks. This paper proposes a homologous multi-point warping for 3D facial landmarking, which is verified experimentally on each of the target objects in a given dataset using 500 landmarks (16 anatomical fixed points and 484 sliding semi-landmarks). This is achieved by building a template mesh as a reference object and applying this template to each of the targets in three datasets using an artificial deformation approach. The semi-landmarks are subjected to sliding along tangents to the curves or surfaces until the bending energy between a template and a target form is minimal. The results indicate that our method can be used to investigate shape variation for multiple datasets when implemented on three databases (Stirling, FRGC and Bosphorus). 


\section{Landmark-based Homologous Multi-point Warping}

2 Approach to 3D Facial Recognition Using Multiple

3 Datasets

4

5 Olalekan Agbolade ${ }^{1}$, Azree Nazri1* ${ }^{*}$, Razali Yaakob ${ }^{1}$, Abdul Azim Abd Ghani ${ }^{2}$, Yoke Kqueen

6 Cheah $^{3}$

7

$8{ }^{1}$ Department of Computer Science, Faculty of Computer Science \& IT, Universiti Putra

9 Malaysia, Selangor, Malaysia

$10{ }^{2}$ Department of Software Engineering, Faculty of Computer Science \& IT, Universiti Putra

11 Malaysia, Selangor, Malaysia

$12{ }^{3}$ Department of Biomedical Science, Faculty of Medicine and Health Sciences, Universiti Putra

13 Malaysia, Selangor, Malaysia

Corresponding Author:

16 Azree Nazri*

17 Email address: azree@upm.edu.my

\section{Abstract}

Over the years, neuroscientists and psychophysicists have been asking whether data acquisition for facial analysis should be performed holistically or with local feature analysis. This has led to various advanced methods of face recognition being proposed, and especially techniques using facial landmarks. The current facial landmark methods in 3D involve a mathematically complex and time-consuming workflow involving semi-landmark sliding tasks. This paper proposes a homologous multi-point warping for 3D facial landmarking, which is verified experimentally on each of the target objects in a given dataset using 500 landmarks (16 anatomical fixed points and 484 sliding semi-landmarks). This is achieved by building a template mesh as a reference object and applying this template to each of the targets in three datasets using an artificial deformation approach. The semi-landmarks are subjected to sliding along tangents to the curves or surfaces until the bending energy between a template and a target form is minimal. The results indicate that our method can be used to investigate shape variation for multiple datasets when implemented on three databases (Stirling, FRGC and Bosphorus).

Human facial traits play an essential role in human identification. The face contains the most important sensory organs and acts as the central interface for appearance, communication, expression and mutual identification (Peng et al. 2013). Landmark-based geometric morphometric 
38

39

40

41

42

43

44

45

46

47

48

49

50

51

52

53

54

55

56

57

58

59

60

61

62

63

64

65

66

67

68

69

70

71

72

73

74

75

76

77

methods for face recognition provide new insights into patterns of biological shape variation that cannot be evaluated by traditional methods (Anies et al. 2013).

Recently, many two-dimensional face recognition systems have been developed, with good results for image acquisition under favorable conditions (Zhao et al. 2003). The major constraints include illumination and changes in pose. In addition to variation due to pose and illumination, which affect $2 \mathrm{D}$ face data, 3D faces are more easily detected due to a higher intensity modality compared with 2D faces (Savran et al. 2012). Furthermore, when they are subjected to systematically increasing pitch and yaw rotation, as shown by Wang Jun (2006), there is a drop in performance related to expression recognition in $2 \mathrm{D}$, while that in $3 \mathrm{D}$ remains constant. This is a result of occlusion effects from substantial distortion in out-of-plane rotations. In addition, in regard to feature transformation and classification, the 3D modality shows some improvement over 2D with a high level of confidence. However, for depth features, both show the same performance. The processing cost of 3D models is higher than that of 2D models (Savran et al. 2012).

The term 'morphometrics' was coined more than 50 years ago by Robert E. Blackith, who applied multivariate statistical methods to the basic carapace morphology of grasshoppers (Elewa \& Elewa 2010). Morphometrics is the study of shape variation and its covariation with other variables (Bookstein 1997a; Dryden \& Mardia 1998). According to Adams et al. (2004), the term traditionally referred to the application of multivariate statistical analyses to sets of quantitative variables such as length, width, height and angle. Advances in morphometrics have shifted the focus to the Cartesian coordinates of anatomical points that can be used to define more traditional measurements. Morphometrics considers variation and group differences in shape, central tendency, and the association of shape with extrinsic factors. This is directly based on the digitized $(\mathrm{x}, \mathrm{y}, \mathrm{z})$-coordinate positions of landmarks, or points representing the spatial positions of putatively homologous structures in 2D or 3D. In contrast, conventional morphometric studies utilize distances as variables (Bookstein 1997a; Dryden \& Mardia 1998; Rohlf 1993).

The thin-plate spline (TPS) is simply a convenient function for capturing changes in landmark configurations and displaying the differences on the smoothest possible transformation grid (Rohlf et al. 1996). This ensures that the points of the starting and target form appear precisely in their corresponding positions in relation to the transformed and untransformed grids (Bookstein 1989). With the application of the iterative closest point (ICP), landmark correspondence can be iteratively registered in the vicinity of a landmark with a re-weighted error function. In work by Wan et al. (2010), a thin-plate spline (TPS) was used to align the points in each facial image. These authors employed ICP to build a correspondence by taking the closest point on each surface mesh, while the inverse of the TPS warp was used to map each surface back to its reference location. The smoothness or point relaxed in TPS was approached based on the minimization of bending energy. This approach computes the amount of deformation between two shape configurations, as quantified by the TPS function through the integral of the squared second derivatives of that deformation (Mitteroecker \& Gunz 2009). The geometric morphometrics (GM) of the Procrustes 
78

79

80

81

82

83

84

85

86

87

88

89

90

91

92

93

94

95

96

97

98

99

100

101

102

103

104

105

106

107

108

109

110

111

112

113

114

115

116

117

118

superimposition method is a least-squares oriented method involving translation, scaling and rotation (Mitteroecker \& Gunz 2009). Shape is the geometric information of an object after the removal of location, orientation and scale (Kendall 1977).

The use of GM has revolutionized the sophistication of the collection and quantitative analysis of biological shapes. It has been applied to solve various research questions relating to plants, animals and humans. Examples include Neanderthal fossils (Rosas et al. 2015), flower shapes (van der Niet et al. 2010), dinosaurs (Fearon \& Varricchio 2015), butterfly wings (Chazot et al. 2016), zebrafish skeletogenesis (Aceto et al. 2015) and humans (Solon 2012; Lindner et al. 2016).

A landmark was defined by Marcus et al. (1993) as a point in a 2D or 3D space that corresponds to the position of a particular trait in an object. Dryden \& Mardia (1998) also described landmarks as points of correspondence on each object that match within and between populations. This set of points, one on each form, are operationally defined for an individual based on local anatomical features, and must be consistent with some hypothesis of biological homology. Bookstein (1997a) and Dryden and Mardia (1998) categorized landmarks into three types: Types I, II and III. Type I landmarks are defined as discrete juxtapositions of tissues such as at the intersection of three sutures, for example the dacryon and asterion (Lynch et al. 1996; Slice 2006) or the bregma and lambda (Bookstein 1997b). Type II landmarks are curvature maxima or other local morphogenetic processes, usually with a biomechanical implication such as a muscle attachment site (Ross \& Williams 2008), for example the prosthion, ectoconchion (Lynch et al. 1996; Slice 2006), subnasale, meatus or nasion (Bookstein 1997b). Type III landmarks are extremal points such as the endpoints of maximum cranial length and breadth (Lynch et al. 1996; Slice 2006) and the orbitale, gonion, glabella and gnathion (Bookstein 1997b).

Facial landmarking is a crucial step in facial analysis for biometrics and numerous other applications. Since 3D data contain more information and are less sensitive to illumination and occlusion than 2D data, the use of 3D data to improve facial analysis is increasing in computer vision (Chen et al. 2015). Many studies of population variation have been performed in morphometric research using facial landmarks. Some investigations have covered certain regions of the face, while some have examined the entire facial region. For instance, significant differences in the symmetric shape component in the nasal region between German and Chinese populations were identified by Schlager and Rüdell (2015), using a dense set of semi-landmarks. An investigation of nose profile morphology in Scottish and Indonesian populations was also carried out by Sarilita et al. (2018), with the aim of improving the accuracy of forensic craniofacial reconstruction. The soft-tissue facial form of high-resolution 3D images of Han Chinese, Tibetan, Uyghur and European people was analyzed by Guo et al. (2014). Facial diversity was examined by establishing a high-density alignment across all faces, and the analyses revealed that the brow area, cheekbones, and nose exhibited strong signals of differentiation between populations. Statistically significant face shape differences between the Dutch and UK population were investigated by Hopman et al. (2014). Mean face shape was visualized using signature heatmap and dynamic morphs, showing that genetic variants influence normal facial variation. Although these studies 
119 examined variation in diverse populations, these populations were drawn from the same datasets.

120

121

122

123

124

125

126

127

128

129

130

131

132

133

134

135

136

137

138

139

140

141

142

143

144

145

146

147

148

149

150

151

152

153

154

155

156

157

158

In contrast, the current study analyzes variation using different datasets.

Since wide biological variability cannot be assessed using only anatomical landmarks (BottonDivet et al. 2015), in order to quantify complex shapes, sliding semi-landmarks have been developed which can be placed on surfaces (Gunz et al. 2005) or curves (Bookstein 1997a; Gunz et al. 2005). This approach generates landmarks that are spatially homologous after sliding (Parr et al. 2012) and can be optimized by minimizing the bending energy (Cornette et al. 2013; Fabre et al. 2014) or Procrustes distance (Perez et al. 2006; Mitteroecker 2013). Several software packages are currently available to perform the sliding of landmarks in 3D, for example Edgewarp (Bookstein \& Green 1994), the EVAN toolbox (http://evan-society.org), Viewbox (Halazonetis 2014), Mathematica (Mitteroecker 2013), and the geomorph (Adams \& Otárola-Castillo 2013) and Morpho (Schlager 2013) R packages.

The aim of this study is to apply the computational deformation process reported by Bookstein (1989). When projecting a surface semi-landmark from the template object to the target object and iteratively sliding the semi-landmark to a point relaxed, the simpler workflow allows us to perform this task in Viewbox 4.0, unlike the complex workflow presented by Botton-Divet et al. (2015). Secondly, this method is not new in terms of analyzing shape variation in morphometric geometry, but its application to the analysis of shape variation for soft-tissue faces in 3D for multiple human datasets is novel. Figure 1 shows a schematic conceptual diagram of the homologous multi-point warping algorithm.

Figure 1: Schematic conceptual diagram of the proposed homologous multi-point warping algorithm

\section{Materials \& Methods}

The use of 3D face images in morphometrics not only gives us scope to cover a wider area of the human facial region, but also retains all the geometric information of the object descriptors (Bookstein 1997b; Dean 1996). Here, our method uses 3D facial images from three different datasets, and uses a morphometric approach to propose a less mathematically complex yet robust algorithm for facial landmarks in 3D.

\section{Dataset \& Description}

We used three datasets to validate the robustness of our method. The first was acquired from the Stirling/ESRC 3D face database, which was captured by a Di3D camera system (Stirling-ESRC 2018). These images are in the format of wavefront obj files containing 101 subjects with $3 \mathrm{D}$ facial scans in a neutral position. The database was intended to facilitate research into face recognition, expression and perception, and we randomly selected 58 subjects for this study. The dataset was used as a test set for a competition involving 3D face reconstruction from $2 \mathrm{D}$ images, with the $3 \mathrm{D}$ scans acting as the 'ground truth', at an IEEE conference. The second dataset was the Bosphorus

Peer] Comput. Sci. reviewing PDF | (CS-2019:01:34630:3:0:CHECK 21 Nov 2019) 
159

160

161

162

163

164

165

166

167

168

169

170

171

172

173

174

175

176

177

178

179

180

181

182

183

184

185

186

187

188

189

190

191

192

193

194

195

196

197

database, which was intended for research on 3D and 2D human face processing tasks and contains 105 subjects. We randomly selected 57 subjects for this study. The dataset was acquired using structured-light-based 3D system, with the subjects being instructed to sit at a $1.5 \mathrm{~m}$ distance from the camera; the sensor resolution in the $\mathrm{x}, \mathrm{y}$ and $\mathrm{z}$ directions was $0.3,0.3$, and $0.4 \mathrm{~mm}$, respectively, and high-resolution color texture was used (Savran et al. 2008). The third dataset was the Face Recognition Grand Challenge Version 2.0 (FRGC v2) database, consisting of 466 facial images, of which we randomly selected 120 subjects for this study. Each subject image was captured under uniform illumination, with high resolution and fairly uncontrolled conditions (Phillips et al. 2005).

\section{Template Mesh}

Figure 2: A 3D mesh template with the location of the prominent point at the center of the face for pose-invariant correction. The 16 fixed anatomical landmarks are shown in red, while the blue area on the pronasale indicates the point at which the semi-landmarks begin the sliding process.

A template mesh of 92,995 vertices and 183,996 triangles was created by manually locating 16 anatomical points on the 3D face (Fig. 2) called fixed points, according to facial landmark standards (Caple \& Stephan 2016) (for more detail, see Table 1). The fixed landmarks were not subjected to sliding, but were used to establish the warping fields for minimizing the bending energy. Due to its ease of detection and pose correction (Creusot et al. 2010) and its invariance to facial expression (Colombo et al. 2006), the nose tip (pronasale) was selected as the most robust and prominent landmark point. The nose tip area can be approximated as a hemisphere on the human face, although any other facial anatomical point could be used. This is where the sliding points begin to spread across the facial surface. Using this fixed point (the pronasale), 484 semilandmarks were automatically generated, with the overlapping on the pronasale shown in blue. These were first randomly placed on the facial mesh before being uniformly distributed on the selected facial surface (Fig. 3). This was done using the locational positions of the fixed anatomical points with $1.5 \mathrm{~mm}$ radius to accommodate all 500 points. To quantify the morphological data for the complex 3D traits of both reference and target shapes, we used geometric morphometric tools based on previously reported landmark-based methodologies (Halazonetis 2014; Klingenberg \& Zaklan 2000; Kouli et al. 2018; Yong et al. 2018; Zelditch et al. 2012), and the automatic point placement, semi-landmark sliding, and landmark acquisition were implemented in ViewBox 4.0 (Halazonetis 2014).

Figure 3: A 3D mesh template of the reference model with 500 landmarks, showing 16 fixed anatomical points and 484 semi-landmarks with $1.5 \mathrm{~mm}$ radius: (A) frontal skewed view; (B) profile view.

Peer] Comput. Sci. reviewing PDF | (CS-2019:01:34630:3:0:CHECK 21 Nov 2019) 
198 Table 1: Fixed anatomical landmarks and descriptions

\section{Homologous Multi-point Warping}

200 The geometry of curves and surfaces is easy in 2D or 3D, but it is less easy to define semi-

201

202

203

204

205

206

207

208

209

210

211

212

213

214

215

216

217

218

219

220

221

222

223

224

225

226

227

228

229

230

231

232

233 landmarks for non-planar surfaces in 3D (Ghislanzoni et al. 2017) as they are not guaranteed to be homologous after the first placement. However, this could be achieved by subjecting the semilandmarks to sliding in the direction that reduces shape variance, thus closely positioning the points at the same locations in the 3D space. The sliding step is important, as it places the landmarks in positions where they correspond better to each other between individuals (Mitteroecker 2013). These semi-landmarks were allowed to slide on the curves and the surface mesh of each target using TPS warping of the template, which positioned the reference points on the target facial mesh by minimizing the bending energy.

According to Bookstein (1989), physical steel takes a bending form with a small displacement. This is because the function $(x, y, z)$ is the configuration of lowest physical bending energy, which is consistent with the given constraints. In this $3 \mathrm{D}$ face deformation, the transformation of TPS is done mathematically via the interpolation of a smooth mapping of $h$ from $\mathbb{R}^{3} \rightarrow \mathbb{R}^{3}$. This is a selected set of corresponding points $\left\{\mathrm{P}_{R i}, \mathrm{P}_{T i}\right\}, i=1, \ldots, N$ on the faces of the reference object (template) and target (subject) that minimizes the bending energy function $\mathrm{E}(h)$ using the following interpolation conditions (Bookstein 1989; Bookstein 1997a; Corner et al. 1992):

$$
\begin{aligned}
& \mathrm{E}(h)=\iiint_{\mathbb{R}^{3}} 3\left(\frac{\partial^{2} h}{\partial x^{2}}\right)^{2}+\left(\frac{\partial^{2} h}{\partial y^{2}}\right)^{2}+\left(\frac{\partial^{2} h}{\partial z^{2}}\right)^{2}+ \\
& \left.2\left(\frac{\partial^{2} h}{\partial x y}\right)^{2}+2\left(\frac{\partial^{2} h}{\partial x z}\right)^{2}+2\left(\frac{\partial^{2} h}{\partial y z}\right)^{2}\right) d x d y d z, \\
& \text { s.t. } \quad h\left(\mathrm{P}_{T i}\right)=\mathrm{P}_{R i}, i=1, \ldots, M
\end{aligned}
$$

where $\mathrm{P}_{T i}$ is the target object, $\mathrm{P}_{R i}$ is the reference object for the sets of corresponding points, and $h$ is the bending energy function that minimizes the non-negative quantity of the interpolation of the integral bending norm or the integral quadratic variation $\mathrm{E}(h)$. The TPS method now decomposes each component into affine and non-affine components, such that

$$
h\left(\mathrm{P}_{h}\right)=\Psi\left(\mathrm{P}_{h}\right) \mathrm{K}+\mathrm{P}_{h} \Gamma
$$

where $\mathrm{P}_{h}$ are the homogeneous coordinate points on the target $3 \mathrm{D}$ face, and $\Psi\left(\mathrm{P}_{h}\right)=\Psi_{1}\left(\mathrm{P}_{h}\right), \Psi_{2}$ $\left(\mathrm{P}_{h}\right), \ldots, \Psi_{M}\left(\mathrm{P}_{h}\right)$ is a $1 \times \mathrm{M}$ kernel vector of TPS of the form:

$\Psi_{w}\left(\mathrm{P}_{h}\right)=\left\|\mathrm{P}_{h}-\mathrm{P}_{T w}\right\|$

$\mathrm{K}$ is a $\mathrm{M} \times 4$ non-affine warping coefficient matrix, and $\Gamma$ is a homogeneous affine transformation of a $4 \times 4$ matrix. The energy function is minimized to find the optimum solution to Eq. (4) if the interpolation condition in Eq. (1) is not met.

$$
\mathbb{E}(\beta, K, \Psi)=\frac{1}{M} \Sigma_{J=1}^{M}\left\|h\left(\mathrm{P}_{T j}\right)-\mathrm{P}_{R j}\right\|+\beta E(h)
$$

The interpolation conditions in Eq. (1) are satisfied if the smoothing regularization term $\beta$ is zero, where $\Gamma$ and $\mathrm{K}$ are TPS parameters obtained by solving the linear equation: 
234

235

236

237

238

239

240

241

242

243

244

245

246

247

248

249

250

251

252

253

254

255

256

257

258

259

260

261

262

263

264

265

266

267

268

269

$$
\left(\begin{array}{cc}
\Psi & \mathrm{P}_{R} \\
\mathrm{P}_{R}^{T} & 0
\end{array}\right)\left(\begin{array}{l}
K \\
\Gamma
\end{array}\right)=\left(\begin{array}{c}
\mathrm{P}_{T} \\
0
\end{array}\right)
$$

$\Psi$ is a $\mathrm{M} \times \mathrm{M}$ matrix with components $\Psi_{w l}=\left\|\mathrm{P}_{T w}-\mathrm{P}_{T l}\right\|$ and $\mathrm{P}_{R}$ is a $\mathrm{M} \times 4$ matrix in which each row is the homogeneous coordinate of the point $\mathrm{P}_{R i}, i=1, \ldots, M$. Using Eq (2), the target facial mesh $\mathrm{P}_{T i}$ was deformed to the reference mesh $\mathrm{P}_{R i}$. The bending energy was applied, and the process was iterated for six cycles to achieve optimum sliding of the points on the facial surface which gives points relaxed. This changed the bending energy from the initial value $E_{i}$ to the final value $E_{f}$ after six complete iterations. This means that the semi-landmarks can be treated in the same way as homologous landmarks in downstream analyses. Since warping may result in points that do not lie directly on the facial surface on the target mesh, the transferred points were projected onto the closest point on the mesh surface using the ICP method (Creusot et al. 2010). The aim of using ICP is to iteratively minimize the mean square error between two point sets. If the distance between the two points is within an acceptable threshold, then the closest point is determined as the corresponding point (Mian et al. 2008). The homologous landmark warping $H_{K \Gamma}$ after six complete iterations is therefore:

$$
H_{K \Gamma}=E_{f-i}\left(\begin{array}{l}
K \\
\Gamma
\end{array}\right)
$$

where

$$
\left(\begin{array}{l}
K \\
\Gamma
\end{array}\right)=\left(\begin{array}{cc}
\Psi & \mathrm{P}_{R} \\
\mathrm{P}_{R}^{T} & 0
\end{array}\right)-1\left(\begin{array}{c}
\mathrm{P}_{T} \\
0
\end{array}\right)
$$

is the linear TPS equation obtained during deformation of the surface of the target mesh to the reference mesh, before convergence was finally reached, and $E_{f-i}=E_{f}-E_{i}$ after six complete iterations. The first iteration showed a partial distribution of sliding points on the target surface mesh (Fig. 4A). This was automatically repeated until the optimum homologous result was achieved, using an exponential decay sliding step of hundred to five percent. During relaxation of the spline, the semi-landmarks were slid along the surface and the curve tangent structures, rather than on the surfaces or the curves. This reduced the computational effort, as the minimization problem became linear since sliding along the tangents lets the semi-landmarks slip off the data (Gunz et al. 2005). The target surface mesh was then treated as a set of homologous points (Fig. 4B). Note that we did not construct a new deformable mathematical equation from scratch, but simply extended the standard deformable method established by Bookstein (1989). We added a minor extension to the computational process of projecting the surface semi-landmark from the template object to the target object and iteratively sliding the semi-landmark to a point relaxed. After applying the step-by-step methods of digitization of the facial points and sliding of the semi-landmarks using ViewBox 4.0, we then applied Procrustes superimposition, error assessment with Procrustes ANOVA, canonical variate analysis, regression analysis, and shape visualization and variation with PCA, using MorphoJ 1.06d (Klingenberg 2011). Boxplots for the size distribution and a MANOVA were performed in PAST 3.0 (Hammer et al. 2001).

PeerJ Comput. Sci. reviewing PDF | (CS-2019:01:34630:3:0:CHECK 21 Nov 2019) 
270 Figure 4: Sliding point warped on the surface of the target face: (A) partial sliding on target

271

272

273

274

275

276

277

278

279

280

281

282

283

284

285

286

287

288

289

290

291

292

293

294

295

296

297

298

299

300

301

302

303

304

305

306

307

308

309

mesh; (B) complete and homologous warping on target mesh.

\section{Sliding Task Comparison}

In many studies dealing with sliding semi-landmarks, many iterations and tasks are required before optimum smoothness is reached, even with a small set of landmarks. Botton-Divet et al. (2015) compared Edgewarp (Bookstein \& Green 1994) and Morpho (Schlager 2013) in terms of the time, workflow complexity, and computational efficiency required to slide semi-landmarks on long bones in different mustelids. The study used 27 manually placed 3D anatomical landmarks and 790 sliding semi-landmarks on curves and surfaces. Our study does not consider the time factor, due to the challenges arising from the different datasets and samples used. Furthermore, many studies that have implemented sliding semi-landmarks have not reported information on the sliding times, giving no scope for comparison. In our study, both the digitization of fixed landmarks and the sliding of semi-landmarks were performed in Viewbox. Unlike in Edgewarp, where digitization was first performed using a Breuckmann 3D white light fringe surface scanner (Botton-Divet et al. 2015), the remaining holes were filled and edges and spikes were removed using Geomagic. It should be noted that the task of surface pre-processing is not required in Viewbox, unlike in Edgewarp, where surface pre-processing, initial projection, and iterations against the Procrustes consensus must be accounted for (Botton-Divet et al. 2015).

\section{Measurement Error}

The process of landmark coordinate extraction is always associated with some degree of measurement error, and this may be as a result of the non-coplanarity of landmarks, inconsistency of specimens relative to the plane of digitization, or difficulties in pinpointing the landmark locus [56]. Landmark digitization error can be minimized by careful landmark selection, but can never be totally eliminated. In assessing measurement error, three templates were designed for each population. The same individuals (five per dataset) were acquired three times each, using the three templates [57-59]. These were digitized for both manual and sliding semi-landmarks on the different reference objects, and this was followed by Procrustes superimposition on the landmark data using three partitions: fixed anatomical landmarks (FAL), sliding semi-landmarks (SSL), and combined landmarks (CL). Although several other error measurement methods were suggested by Fruciano (2016), the measurement error for this study was assessed using a Procrustes ANOVA. This technique (Klingenberg et al. 2002; Klingenberg \& McIntyre 1998) was implemented in morphometrics to analyze measurement errors (Klingenberg et al. 2010; Leamy 2015; Singh et al. 2012) using MorphoJ, which was achieved through the minimization of the squared sum of the distances of all objects and the consensus configuration (Fruciano 2016). This approach uses a partition based on the sum of squares of the deviations from the average configuration of each coordinate in a two-factor ANOVA, which can then be summed across all the coordinates. Using a relevant number of degrees of freedom, computation of the mean squares is done by dividing the total sum of squares for an effect (Klingenberg \& McIntyre 1998). The Procrustes approach is

PeerJ Comput. Sci. reviewing PDF | (CS-2019:01:34630:3:0:CHECK 21 Nov 2019) 
310

311

312

313

314

315

316

317

318

319

320

321

322

323

324

325

326

327

328

329

330

331

332

333

334

335

336

337

338

339

340

341

\section{2}

343

344

345

346

347

most useful in computing mean shapes and in deciding whether two shapes $\left[R_{i}\right]$ and $\left[T_{i}\right]$ are random realizations of the same shape (Goodall 1991).

The steps in this algorithm can be summarized as follows:

1. Anatomical fixed points (16) were digitized on the template facial mesh and a prominent point (the pronasale) was identified.

2. Semi-landmarks (484) were automatically generated and placed along the curves, located at a uniform distance along each curve for sliding in Step 5.

3. These semi-landmarks were first randomly placed and then uniformly distributed on the selected reference surface mesh, starting from the selected prominent point.

4. The reference facial model was warped to each target mesh configuration using a TPS transformation, and the surface semi-landmark was projected from the reference facial mesh to the target facial mesh.

5. The surface and curve semi-landmarks were then slid together in the direction that minimized the bending energy between each target configuration and the reference object. This was done iteratively in six complete cycles, in order to ensure convergence and optimum smoothness. This gave a homologous representation of the reference mesh.

6. A Procrustes superimposition of the landmark data was performed, and an error assessment was computed using a Procrustes ANOVA.

\section{Shape and Size Variation}

Since there may be an interaction between the size and shape in facial morphology due to changes in the shape associated with size differences [62], we assessed the allometry by testing the statistical significant proportion of morphological variation in the shape components, using a multivariate regression of shape onto size. Canonical variate analysis (CVA) was also performed to test the group differences and an ordination plot was produced (plot not shown).

Differences in the effects and size were then examined by computing a non-parametric analysis of variance (MANOVA) in terms of Wilks' lambda. Using the population as the group and the size as the covariate, the population by size interaction term was calculated. The MANOVA was recomputed after removing the interaction term (population by size) and the population effect tested for the difference in the regression intercept.

\section{Results}

\section{Sliding Task}

The tasks required for the initial projection and the relaxations against the Procrustes mean shape for the iterations are minimal in Viewbox, which requires a much simpler workflow compared to Edgewarp. Moreover, since a general Procrustes analysis was required in order to perform the sliding task, which is not implemented internally in Edgewarp, new input files were generated prior 
348 to performing the next iteration (Botton-Divet et al. 2015), creating greater complexity in the 349 workflow.

350

351

352

353

354

355

356

357

358

359

360

361

362

363

364

365

366

367

368

369

370

371

372

373

374

375

376

377

378

379

380

381

382

383

384

385

386

387

\section{PCA}

After calculating the mean shape using Procrustes superimposition, we studied the variability in the shape using PCA. This was not only to reduce the number of dimensions but also to balance the fit of the model with the ease of analysis and potential loss of information (Cangelosi \& Goriely 2007). The PCA of the total sample yielded 168 principal components. When they were separately computed, the Stirling population yielded only 57 PCs, FRGC yielded 59 PCs, and Bosphorus 51 PCs, all with non-zero variability. In order to retain any component that accounted for a specific proportion or percentage, we used a broken stick approach to PCA selection (Cangelosi \& Goriely 2007; Klingenberg 2013; Peres-Neto et al. 2005). The first five PCs accounted for $98.05 \%$ of the total variation in the Stirling population, while the first four PCS accounted for $93.44 \%$ of the total variation in the FRGC population, the first three PCs accounted for $85.65 \%$ of the total variation in the Bosphorus population, and the first two PCs accounted for $97.17 \%$ of the total variation in the combined population (Cangelosi \& Goriely 2007). The first and second PCs (PC1 and PC2) of the Stirling population accounted for $65.40 \%$ and $20.03 \%$ of the variation, respectively; in the FRGC population, these accounted for $76.88 \%$ and $7.97 \%$, respectively; for the Bosphorus population, these figures were $68.29 \%$ and $10.84 \%$, respectively; and for the total population, they were $93.96 \%$ and $3.21 \%$, respectively.

Figure 5: Visualization using 3D dataset objects and lollipop graphs. (A)-(D) represent a 3D dataset object on three axes, showing only axis 1 vs axis 3: (A) Stirling; (B) FRGC; (C) Bosphorus; (D) combined population, with variance contributed by each PC. (E)-(H) show lollipop visualizations of the first principal component of each population and combined population: (E) Stirling; (F) FRGC; (G) Bosphorus; (H) combined population.

Shape variations for each population and the combined population are shown in Figure 5. In the visualization of $3 \mathrm{D}$ dataset object, the number of landmarks is shown in red and the mean symmetry configuration is shown in light blue. A lollipop graph is shown of the first principal component of each population and the combined population, which indicates the difference in the face shape. Note that we only visualized the first PC of each population, since this accounted for the highest variation in the total shape.

\section{Procrustes ANOVA and Shape Variation}

A Procrustes superimposition of each set configuration produced a symmetric consensus configuration. When the variation was partitioned around this consensus using a Procrustes ANOVA (Table 2), the results showed that the variation in symmetric shape among individuals in each set accounted for the largest portion of the total variation, although this was not statistically significant for the manually placed landmarks. To assess the digitization errors of the manually 
388

389

390

391

392

393

394

395

396

397

398

399

400

401

402

403

404

405

406

407

408

409

410

411

412

413

414

415

416

417

418

419

420

421

422

423

424

425

placed landmarks, the sliding semi-landmarks and overall landmarks, the deviations for each were obtained by simply calculating the amount of displacement from the average position calculated from all digitization and the variation accounts for the smallest portion of the total variation. For manually placed (fixed anatomical) landmarks, the variation accounted for $4.31 \%$, while for sliding semi-landmarks, this accounted for $3.71 \%$, and for the overall landmarks, this was $3.67 \%$.

The CVA indicated that each population studied was clearly distinct from the others. The Procrustes distances between populations (FRGC vs. Bosphorus $=0.040$; Stirling vs. Bosphorus $=$ 0.580; Stirling vs. FRGC $=0.061)$ were all statistically significant $(p<0.0001)$. All 10,000 pairwise permutation tests indicated that the mean shapes differed significantly in the population.

Table 2: Procrustes ANOVA for facial shape with digitization errors

Figure 6A shows the first two principal components of the total shape in the three populations, accounting for the total variance. The scatterplots of the scores along the first two principal components for all the datasets (Figure 6A) showed an apparent pattern of association between the manual anatomical landmarks and the sliding semi-landmarks for the same specimen. This pattern disappeared when the sliding semi-landmarks were removed, as the data for Bosphorus and FRGC were clustered more tightly.

Changes in the shape of the face as a function of size (allometry) were evaluated using multivariate regression of the effect of shape variables on the centroid size for all populations (Figure 6B). This is usually of primary interest within a homogeneous population. Inter-population allometry explained only $13.28 \%$ of the shape differences according to size, and was significant ( $p$ $<0.0001)$. The box-plots in Figure 6C showed that the Bosphorus dataset contained the largest sizes, followed by FRGC, while the Stirling dataset contained the smallest sizes.

Figure 6: Principal components, multivariate regression and boxplots for the population groups. (A) Scatterplots of PC1 vs. PC2, which together explain more than 50\% of the variance. Left: manual anatomical landmarks; center: sliding semi-landmarks; right: all landmarks. (B) Allometric regression of population mean shape. (C) Boxplot for centroid size for all populations, showing the size variation after averaging faces within populations.

The characteristics of the allometric trajectories of the population were then tested using a MANOVA (Table 3), which explained a significant proportion of the overall variation. The interaction term (test for slopes) was statistically significant. When the size effect was removed and the MANOVA was repeated, the result was still statistically significant, suggesting that the effect of size on shape for both the slope and intercept was strong, and that this was not the case in the population group.

Table 3: MANOVA results in terms of Wilks' Lambda 


\section{Discussion}

427 The use of landmarks has evolved in terms of locating biological or anatomical features on human

428

429

430

431

432

433

434

435

436

437

438

439

440

441

442

443

444

445

446

447

448

449

450

451

452

453

454

455

456

457

458

459

460

461

462

463

464

465 faces. However, its validity is based on a morphometric analysis, which depends upon the biological justification for the designation of the landmarks, as stated by Bookstein (1997b). In performing the sliding task, Edgewarp appears to be more task-complex than Viewbox. The iterative relaxation in Edgewarp requires several manual operations per iteration, including data saving after sliding (Botton-Divet et al. 2015). This means that the workflow complexity is reduced, and the computational efficiency is higher in Viewbox than in Edgewarp. Although Morpho has been proven to be more efficient, since its functions can often be run on several cores many times, difficult coding is required to perform a single task.

Our analyses demonstrated significant differences in the average shape of the symmetric components across groups and the symmetric components of shape between individuals using the proposed method. The results of the Procrustes ANOVA suggested a modest but nevertheless appreciable variation in shape and size. Shape differences were statistically significant even after averaging faces within populations, and the small measurement errors (MAL $=0.063, \mathrm{SSL}=$ 0.026 , and $C L=0.027$ ) show that the landmarks can be annotated with precision using the proposed method. Sliding semi-landmarks produced a more accurate error result, while manually placed landmarks gave a less accurate error value. This could be a result of difficulty in pinpointing the locus of the landmark (Webster \& Sheets 2010). However, many approaches are available for addressing the measurement error when combining samples from multiple sources. A full discussion of this topic is beyond the scope of this study, but extended details can be found in (Fruciano 2016). Our approach simply allows us to say that when the template used is changed, the shape does not change significantly (compared to other biological and non-biological sources of variation). To investigate allometry, a scatterplot of the regression score was plotted to show the regression of shape onto size pooling within populations (the projection of shapes in the direction of the vector of regression coefficients) vs. centroid size (Drake \& Klingenberg 2007). Box plots of centroid size differ significantly within each group. The centroid size was not log-transformed, as this transformation made no appreciable difference in the results. Despite their significance, the sizes of the effects being tested are small or similar in relative terms, and we must therefore interpret these effects with caution (Daboul et al. 2018). The MANOVA test based on Wilks' Lambda showed a significant result for both slope and intercept $(p<0.000)$. The visualization of face shape was done using PCA after the Procrustes fit of the dataset object; however, we present only axis 1 vs. axis 3, as the other two axes do not represent a normal facial shape. The results of visualization showed that Bosphorus faces have less prognathic faces and dropped chins, the FRCG faces have wide noses and long foreheads, and the Stirling faces have wide noses and wide foreheads.

In general, the face shapes in the Stirling and FRGC dataset are smaller than those in the Bosphorus dataset, and were characterized by geographical locations. This matches our expectations, as we were comparing samples from two neighboring regions and a different region.

PeerJ Comput. Sci. reviewing PDF | (CS-2019:01:34630:3:0:CHECK 21 Nov 2019) 
466 Both the Stirling and FRGC populations were based on the Western hemisphere (the UK and US, 467 respectively) while the Bosphorus population was based on the Eastern hemisphere (Western

468 Asia). Although an investigation of the variation among different data sources could be much more 469 complex and complicated than that presented here, our goal in this study was to make the analysis

470 as simple as possible, and this could be extended in future work. Readers should therefore be aware 471 that the differences found between datasets are at least in part due to differences in the way these 472 datasets were acquired (although it is hard to say how far this applies).

473

\section{Conclusions}

475 This method combines pragmatic solutions for configuring an optimized pipeline for a high-

476

477

478

479

480

481

482

483

484

485

486

487

488

489

490

491

492

493

494

495

496

497

498

499

500

501

502

503

504

505

506 throughput homologous multi-point deformable 3D facial signature. We warped only the reference surfaces and curves to each sample face, using an automatic homologous warping approach. The results of a Procrustes ANOVA show that the measurement error may be a source of substantial variation when combining different morphometric datasets, and may sometimes have an unexpected effect on parameter estimates (Fruciano et al. 2017). High-throughput phenotypic facial data such as these may be valuable in forensic studies of human facial morphology, anthropology, disease diagnosis and prediction, statistical shape or image analysis, face recognition, age estimation, facial-based sex dimorphism, and facial expression recognition. A limitation of the Viewbox is that it only runs on Windows OS, while Edgewarp runs on both Linux and Windows OS.

\section{Acknowledgements}

We would like to thank the Face Recognition Grand Challenge, NIST, Bosphorus (Bogazici University), and Stirling/ESRC (University of Stirling) for prompt agreement to use their datasets, and the Computer Laboratory of the Faculty of Computer Science \& Information Technology, Universiti Putra Malaysia.

\section{References}

Aceto J, Nourizadeh-Lillabadi R, Marée R, Dardenne N, Jeanray N, Wehenkel L, Aleström P, van Loon JJ, and Muller M. 2015. Zebrafish bone and general physiology are differently affected by hormones or changes in gravity. PLoS One 10:e0126928.

Adams DC, and Otárola-Castillo E. 2013. geomorph: an R package for the collection and analysis of geometric morphometric shape data. Methods in Ecology and Evolution 4:393-399.

Adams DC, Rohlf FJ, and Slice DE. 2004. Geometric morphometrics: Ten years of progress following the 'revolution'. Italian Journal of Zoology 71:5-16. 10.1080/11250000409356545

Anies OS, Torres MAJ, Manting MM, and Demayo CG. 2013. Landmark-Based Geometric Morphometrics in Describing Facial Shape of the Sama-Banguingui Tribe from the Philippines. Journal of Medical and Bioengineering 2:131-136. 10.12720/jomb.2.2.131136 
507

508

509

510

511

512

513

514

515

516

517

518

519

520

521

522

523

524

525

526

527

528

529

530

531

532

533

534

535

536

537

538

539

540

541

542

543

544

545

546

547

548

549

550

551

552

553

554

555

556
Barros JMD, Mirbach B, Garcia F, Varanasi K, and Stricker D. 2018. Fusion of Keypoint Tracking and Facial Landmark Detection for Real-Time Head Pose Estimation. Applications of Computer Vision (WACV), 2018 IEEE Winter Conference on: IEEE. $p$ 2028-2037.

Bookstein FL. 1989. Principal warps: Thin-plate splines and the decomposition of deformations. IEEE Trans Pattern Anal Mach Intell 11:567-585.

Bookstein FL. 1997a. Landmark methods for forms without landmarks- morphometrics of group differences in outline shape. Medical Image Analysis 1.

Bookstein FL. 1997b. Morphometric tools for landmark data: geometry and biology: Cambridge University Press.

Bookstein FL, and Green WD. 1994. Edgewarp: A flexible program package for biometric image warping in two dimensions. Visualization in Biomedical Computing 1994: International Society for Optics and Photonics. p 135-147.

Botton-Divet L, Houssaye A, Herrel A, Fabre A-C, and Cornette R. 2015. Tools for quantitative form description; an evaluation of different software packages for semi-landmark analysis. PeerJ 3:e1417.

Cangelosi R, and Goriely A. 2007. Component retention in principal component analysis with application to cDNA microarray data. Biology direct 2:2.

Caple J, and Stephan CN. 2016. A standardized nomenclature for craniofacial and facial anthropometry. International Journal of Legal Medicine 130:863-879.

Chazot N, Panara S, Zilbermann N, Blandin P, Le Poul Y, Cornette R, Elias M, and Debat V. 2016. Morpho morphometrics: shared ancestry and selection drive the evolution of wing size and shape in Morpho butterflies. Evolution 70:181-194.

Chen L, Mu Z, Zhang B, and Zhang Y. 2015. Ear recognition from one sample per person. PLoS One 10:e0129505. 10.1371/journal.pone.0129505

Christine Cherry E. Solon MAJT, Cesar G. Demayo. 2012. Describing the shape of the face of hypertensive and non-hypertensive adult females using geometric morphometric analysis. International Journal of the Bioflux Society 4.

Colombo A, Cusano C, and Schettini R. 2006. 3D face detection using curvature analysis. Pattern Recognition 39:444-455. 10.1016/j.patcog.2005.09.009

Corner BD, Lele S, and Richtsmeier JT. 1992. Measuring precision of three-dimensional landmark data. J Quant Anthropol 3:347-359.

Cornette R, Baylac M, Souter T, and Herrel A. 2013. Does shape co-variation between the skull and the mandible have functional consequences? A 3D approach for a 3D problem. $J$ Anat 223:329-336.

Creusot C, Pears N, and Austin J. 2010. 3D face landmark labelling. Proceedings of the ACM workshop on 3D object retrieval: ACM. p 27-32.

Daboul A, Ivanovska T, Bülow R, Biffar R, and Cardini A. 2018. Procrustes-based geometric morphometrics on MRI images: An example of inter-operator bias in 3D landmarks and its impact on big datasets. PLoS One 13:e0197675.

Dean D. 1996. Three-dimensional data capture and visualization. Advances in morphometrics: Springer, 53-69.

Drake AG, and Klingenberg CP. 2007. The pace of morphological change: historical transformation of skull shape in St Bernard dogs. Proceedings of the Royal Society B: Biological Sciences 275:71-76.

Dryden IL, and Mardia KV. 1998. Statistical Shape Analysis. Chichester: Wiley.

El Rai MC, Tortorici C, Al-Muhairi H, Werghi N, and Linguraru M. 2016. Facial landmarks detection using 3D constrained local model on mesh manifold. Circuits and Systems (MWSCAS), 2016 IEEE 59th International Midwest Symposium on: IEEE. p 1-4.

Elewa AM, and Elewa AM. 2010. Morphometrics for nonmorphometricians: Springer. 
557

558

559

560

561

562

563

564

565

566

567

568

569

570

571

572

573

574

575

576

577

578

579

580

581

582

583

584

585

586

587

588

589

590

591

592

593

594

595

596

597

598

599

600

601

602

603

604

605

606

607

Fabre AC, Goswami A, Peigné S, and Cornette R. 2014. Morphological integration in the forelimb of musteloid carnivorans. J Anat 225:19-30.

Fearon JL, and Varricchio DJ. 2015. Morphometric analysis of the forelimb and pectoral girdle of the Cretaceous ornithopod dinosaur Oryctodromeus cubicularis and implications for digging. Journal of Vertebrate Paleontology 35:e936555.

Fruciano C. 2016. Measurement error in geometric morphometrics. Dev Genes Evol 226:139158. $10.1007 / \mathrm{s} 00427-016-0537-4$

Fruciano C, Celik MA, Butler K, Dooley T, Weisbecker V, and Phillips MJ. 2017. Sharing is caring? Measurement error and the issues arising from combining 3D morphometric datasets. Ecology and evolution 7:7034-7046.

Goodall C. 1991. Procrustes methods in the statistical analysis of shape. Journal of the Royal Statistical Society Series B (Methodological):285-339.

Gunz P, Mitteroecker P, and Bookstein FL. 2005. Semilandmarks in three dimensions. Modern morphometrics in physical anthropology: Springer, 73-98.

Guo J, Tan J, Yang Y, Zhou H, Hu S, Hashan A, Bahaxar N, Xu S, Weaver TD, and Jin L. 2014. Variation and signatures of selection on the human face. Journal of human evolution 75:143-152.

Halazonetis D. 2014. Viewbox 4 - Cephalometric Software. Available at http://dhal.com/viewboxindex.htm2018).

Hammer $\varnothing$, Harper D, and Ryan P. 2001. Paleontological statistics software: package for education and data analysis. Palaeontologia Electronica.

Hopman SM, Merks JH, Suttie M, Hennekam RC, and Hammond P. 2014. Face shape differs in phylogenetically related populations. European journal of human genetics 22:1268.

Huanca Ghislanzoni L, Lione R, Cozza P, and Franchi L. 2017. Measuring 3D shape in orthodontics through geometric morphometrics. Prog Orthod 18:38. 10.1186/s40510017-0194-9

Kendall DG. 1977. The diffusion of shape. Advances in applied probability 9:428-430.

Klingenberg C, Wetherill L, Rogers J, Moore E, Ward R, Autti-Rämö I, Fagerlund A, Jacobson S, Robinson L, and Hoyme H. 2010. Prenatal alcohol exposure alters the patterns of facial asymmetry. Alcohol 44:649-657.

Klingenberg CP. 2011. MorphoJ: an integrated software package for geometric morphometrics. Molecular Ecology Resources 11:353-357. 10.1111/j.1755-0998.2010.02924.x

Klingenberg CP. 2013. Visualizations in geometric morphometrics: how to read and how to make graphs showing shape changes. Hystrix, the Italian Journal of Mammalogy 24. 10.4404/hystrix-24.1-7691

Klingenberg CP, Barluenga M, and Meyer A. 2002. Shape analysis of symmetric structures: quantifying variation among individuals and asymmetry. Evolution 56:1909-1920.

Klingenberg CP, and McIntyre GS. 1998. Geometric morphometrics of developmental instability: analyzing patterns of fluctuating asymmetry with Procrustes methods. Evolution 52:1363-1375.

Klingenberg CP, and Zaklan SD. 2000. Morphological integration between developmental compartments in the Drosophila wing. Evolution 54:1273-1285.

Kouli A, Papagiannis A, Konstantoni N, Halazonetis DJ, and Konstantonis D. 2018. A geometric morphometric evaluation of hard and soft tissue profile changes in borderline extraction versus non-extraction patients. European journal of orthodontics.

Leamy LJK, Christian Peter; Sherratt, Emma; Wolf, Jason B; Cheverud, James M. 2015. The Genetic Architecture of Fluctuating Asymmetry of Mandible Size and Shape in a Population of Mice: Another Look. Symmetry 7:146-163. 10.3390/sym7010146

Lee MK, Shaffer JR, Leslie EJ, Orlova E, Carlson JC, Feingold E, Marazita ML, and Weinberg SM. 2017. Genome-wide association study of facial morphology reveals novel associations with FREM1 and PARK2. PLoS One 12:e0176566.

Peer] Comput. Sci. reviewing PDF | (CS-2019:01:34630:3:0:CHECK 21 Nov 2019) 
608

609

610

611

612

613

614

615

616

617

618

619

620

621

622

623

624

625

626

627

628

629

630

631

632

633

634

635

636

637

638

639

640

641

642

643

644

645

646

647

648

649

650

651

652

653

654

655

656

657

658

Lindner C, Wang C-W, Huang C-T, Li C-H, Chang S-W, and Cootes TF. 2016. Fully automatic system for accurate localisation and analysis of cephalometric landmarks in lateral cephalograms. Sci Rep 6:33581.

Lynch JM, Wood CG, and Luboga SA. 1996. Geometric morphometrics in primatology: craniofacial variation in Homo sapiens and Pan troglodytes. Folia Primatologica 67:1539.

Marcus LF, Bello E, García-Valdecasas A, and Museo Nacional de Ciencias N. 1993. Contributions to morphometrics: Consejo Superior de Investigaciones Científicas.

Mian AS, Bennamoun M, and Owens R. 2008. Keypoint detection and local feature matching for textured 3D face recognition. International Journal of Computer Vision 79:1-12.

Mitteroecker P, and Gunz P. 2009. Advances in Geometric Morphometrics. Evolutionary Biology 36:235-247. 10.1007/s11692-009-9055-x

Murphy M-M, and Seguchi N. 2019. Digital model sample-Scanning and processing protocol. 3D Data Acquisition for Bioarchaeology, Forensic Anthropology, and Archaeology: Elsevier, 17-45.

Parr W, Wroe S, Chamoli U, Richards H, McCurry M, Clausen P, and McHenry C. 2012. Toward integration of geometric morphometrics and computational biomechanics: new methods for 3D virtual reconstruction and quantitative analysis of Finite Element Models. Journal of Theoretical Biology 301:1-14.

Peng S, Tan J, Hu S, Zhou H, Guo J, Jin L, and Tang K. 2013. Detecting genetic association of common human facial morphological variation using high density $3 \mathrm{D}$ image registration. PLoS computational biology 9:e1003375.

Peres-Neto PR, Jackson DA, and Somers KM. 2005. How many principal components? Stopping rules for determining the number of non-trivial axes revisited. Computational Statistics \& Data Analysis 49:974-997.

Perez SI, Bernal V, and Gonzalez PN. 2006. Differences between sliding semi-landmark methods in geometric morphometrics, with an application to human craniofacial and dental variation. J Anat 208:769-784.

Philipp Mitteroecker PG, Sonja Windhagerc, Katrin Schaefer. 2013. A brief review of shape, form, and allometry in geometric morphometrics, with applications to human facial morphology. Hystrix, the Italian Journal of Mammalogy. 10.4404/hystrix-24.1-6369

Phillips PJ, Flynn PJ, Scruggs T, Bowyer KW, Chang J, Hoffman K, Marques J, Min J, and Worek W. 2005. Overview of the face recognition grand challenge. Computer vision and pattern recognition, 2005 CVPR 2005 IEEE computer society conference on: IEEE. $p$ 947-954.

Rohlf FJ. 1993. Relative warp analysis and an example of its application to mosquito. Contributions to morphometrics 8:131.

Rohlf FJ, Loy A, and Corti M. 1996. Morphometric analysis of Old World Talpidae (Mammalia, Insectivora) using partial-warp scores. Systematic Biology 45:344-362.

Rosas A, Pérez-Criado L, Bastir M, Estalrrich A, Huguet R, García-Tabernero A, Pastor JF, and De la Rasilla M. 2015. A geometric morphometrics comparative analysis of Neandertal humeri (epiphyses-fused) from the El Sidrón cave site (Asturias, Spain). Journal of human evolution 82:51-66.

Ross AH, and Williams S. 2008. Testing repeatability and error of coordinate landmark data acquired from crania. Journal of forensic sciences 53:782-785.

Sarilita E, Rynn C, Mossey PA, Black S, and Oscandar F. 2018. Nose profile morphology and accuracy study of nose profile estimation method in Scottish subadult and Indonesian adult populations. International Journal of Legal Medicine 132:923-931.

Savran A, Alyüz N, Dibeklioğlu H, Çeliktutan O, Gökberk B, Sankur B, and Akarun L. 2008. Bosphorus database for 3D face analysis. European Workshop on Biometrics and Identity Management: Springer. p 47-56.

Peer] Comput. Sci. reviewing PDF | (CS-2019:01:34630:3:0:CHECK 21 Nov 2019) 
659

660

661

662

663

664

665

666

667

668

669

670

671

672

673

674

675

676

677

678

679

680

681

682

683

684

685

686

687

688

689

690

691

692

693

694

695

696

697

698

Savran A, Sankur B, and Bilge MT. 2012. Comparative evaluation of 3D vs. 2D modality for automatic detection of facial action units. Pattern Recognition 45:767-782.

Schlager S. 2013. Morpho: Calculations and visualisations related to Geometric Morphometrics. $R$ package version 0233.

Schlager S, and Rüdell A. 2015. Analysis of the human osseous nasal shape-Population differences and sexual dimorphism. American Journal of Physical Anthropology 157:571-581.

Singh N, Harvati K, Hublin J-J, and Klingenberg CP. 2012. Morphological evolution through integration: a quantitative study of cranial integration in Homo, Pan, Gorilla and Pongo. Journal of human evolution 62:155-164.

Slice DE. 2006. Modern morphometrics in physical anthropology: Springer Science \& Business Media.

Stirling-ESRC. 2018. Stirling-ESRC 3D Face Database. Available at http://pics.stir.ac.uk/ESRC/3d images.htm.

van der Niet T, Zollikofer CP, de León MSP, Johnson SD, and Linder HP. 2010. Threedimensional geometric morphometrics for studying floral shape variation. Trends in plant science 15:423-426.

Vezzetti E, Marcolin F, Tornincasa S, Ulrich L, and Dagnes N. 2017. 3D geometry-based automatic landmark localization in presence of facial occlusions. Multimedia Tools and Applications:1-29. DOI 10.1007/s11042-017-5025-y

Viscosi V, and Cardini A. 2011. Leaf morphology, taxonomy and geometric morphometrics: a simplified protocol for beginners. PLoS One 6:e25630.

Wan J, Shen L, Fang S, McLaughlin J, Autti-Rämö I, Fagerlund Å, Riley E, Hoyme HE, Moore ES, and Foroud T. 2010. A framework for 3D analysis of facial morphology in fetal alcohol syndrome. International Workshop on Medical Imaging and Virtual Reality: Springer. p 118-127.

Wang Jun YL, Wei Xiaozhou, Sun Yi. 2006. 3D facial expression recognition based on primitive surface feature distribution. 2006 IEEE Computer Society Conference on Computer Vision and Pattern Recognition (CVPR'06): IEEE. p 1399-1406.

Webster M, and Sheets HD. 2010. A practical introduction to landmark-based geometric morphometrics. The Paleontological Society Papers 16:163-188.

Yong R, Ranjitkar S, Lekkas D, Halazonetis D, Evans A, Brook A, and Townsend G. 2018. Three-dimensional (3D) geometric morphometric analysis of human premolars to assess sexual dimorphism and biological ancestry in Australian populations. American Journal of Physical Anthropology.

Zelditch ML, Swiderski DL, and Sheets HD. 2012. Geometric morphometrics for biologists: a primer: Academic Press.

Zhao W, Chellappa R, Phillips PJ, and Rosenfeld A. 2003. Face recognition: A literature survey. ACM computing surveys (CSUR) 35:399-458.

Peer] Comput. Sci. reviewing PDF | (CS-2019:01:34630:3:0:CHECK 21 Nov 2019) 
Figure 1

Schematic conceptual diagram of the proposed homologous multi-point warping algorithm

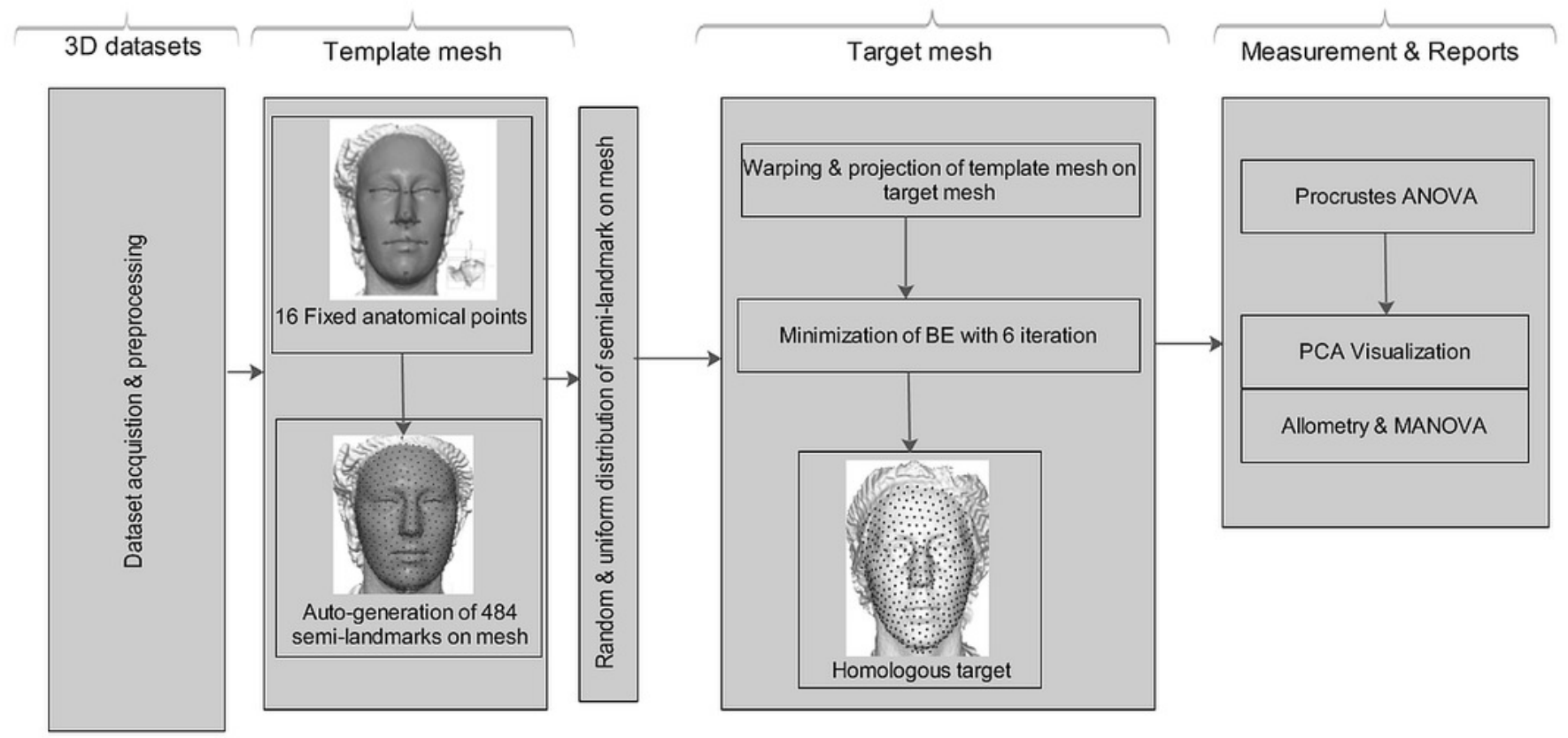




\section{Figure 2}

A 3D mesh template with the location of the prominent point at the center of the face for pose-invariant correction

The 16 fixed anatomical landmarks are shown in red, while the blue area on the pronasale indicates the point at which the semi-landmarks begin the sliding process. 


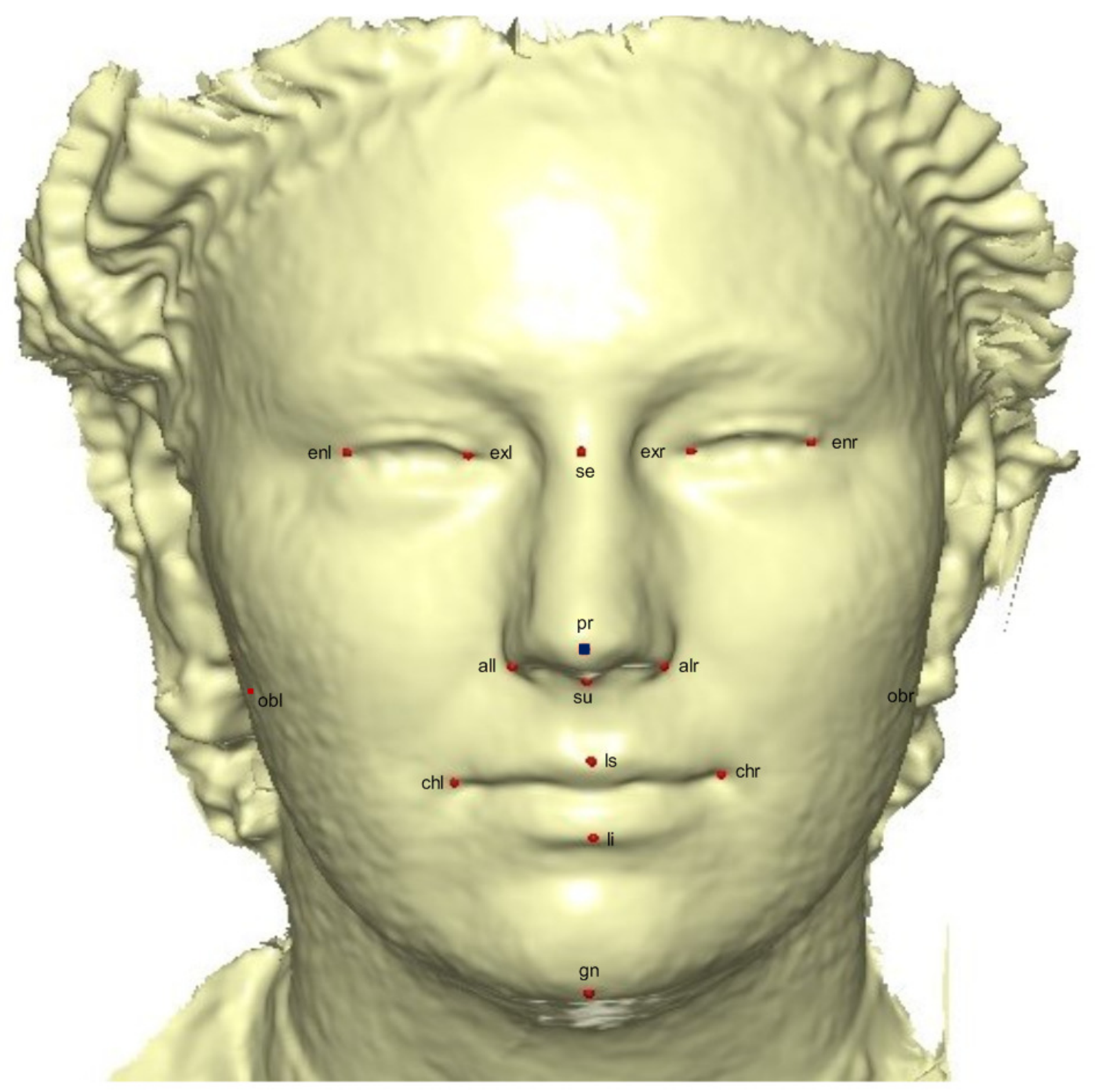


Figure 3

A 3D mesh template of the reference model with 500 landmarks.

Showing 16 fixed anatomical points and 484 semi-landmarks with $1.5 \mathrm{~mm}$ radius: (A) frontal skewed view; (B) profile view.

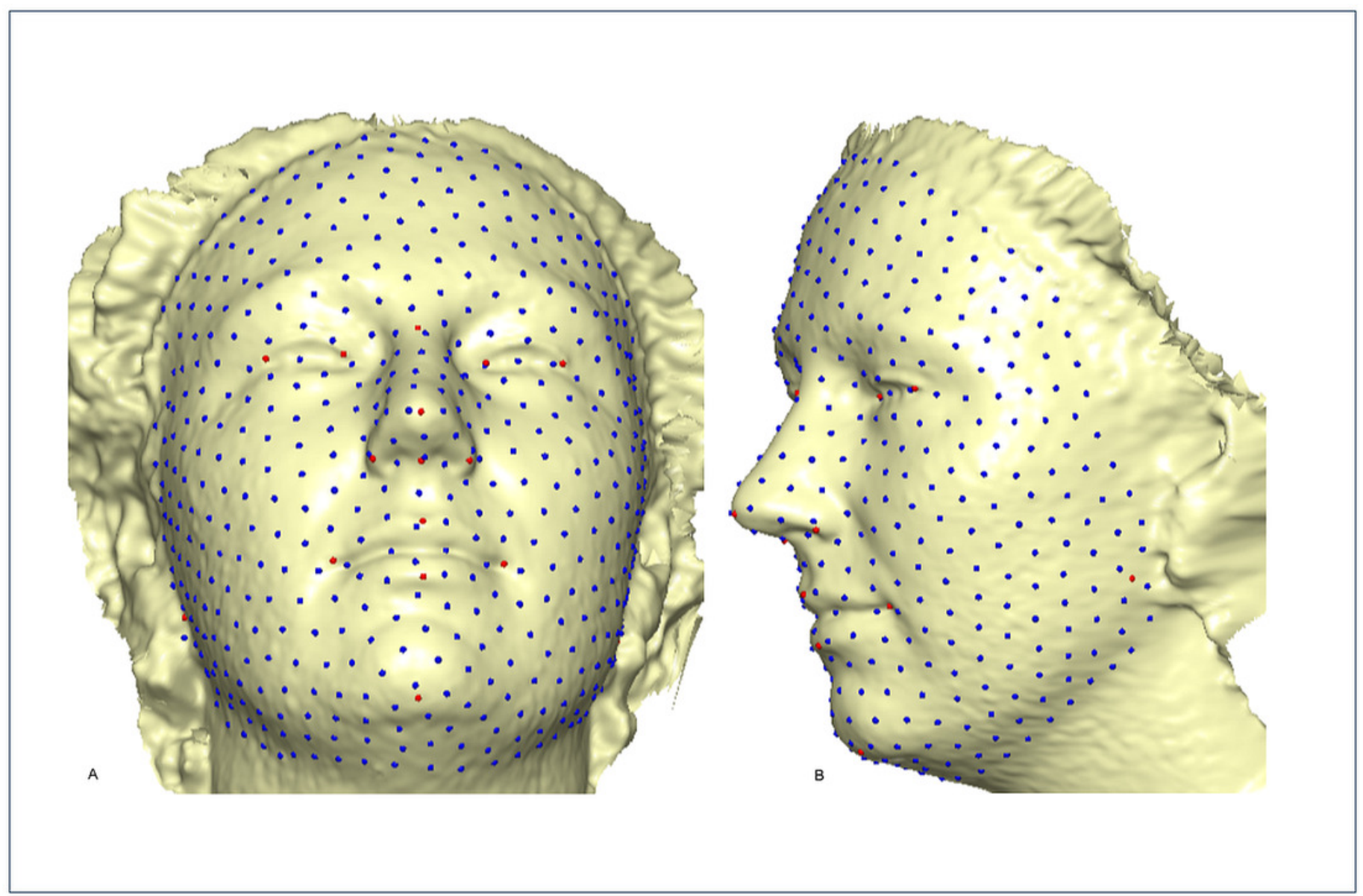


Figure 4

Sliding point warped on the surface of the target face:

(A) partial sliding on target mesh; (B) complete and homologous warping on target mesh.
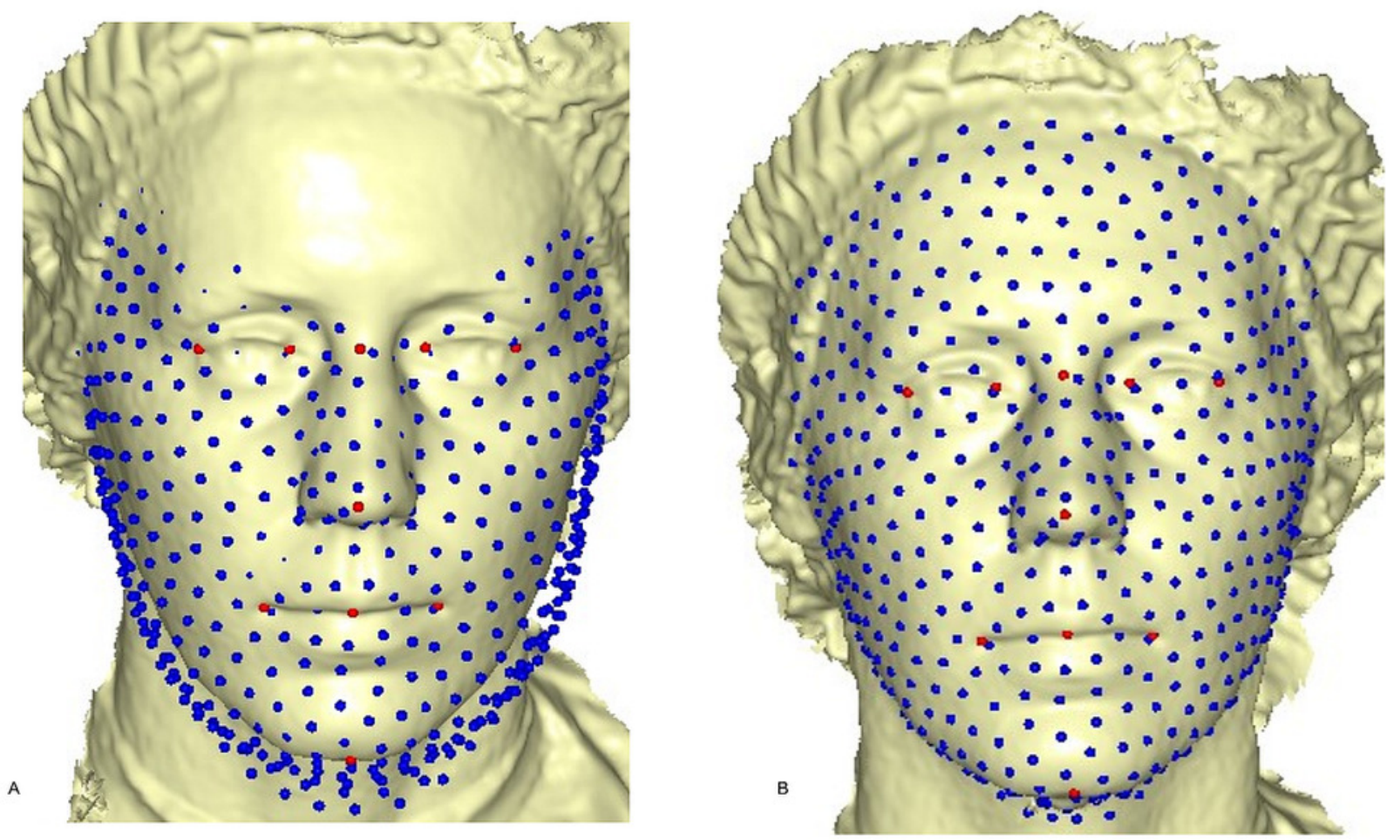


\section{Figure 5}

Visualization using 3D dataset objects and lollipop graphs.

(A)-(D) represent a 3D dataset object on three axes, showing only axis 1 vs axis 3: (A)

Stirling; (B) FRGC; (C) Bosphorus; (D) combined population, with variance contributed by each PC. (E)-(H) show lollipop visualizations of the first principal component of each population and combined population: (E) Stirling; (F) FRGC; (G) Bosphorus; (H) combined population. 


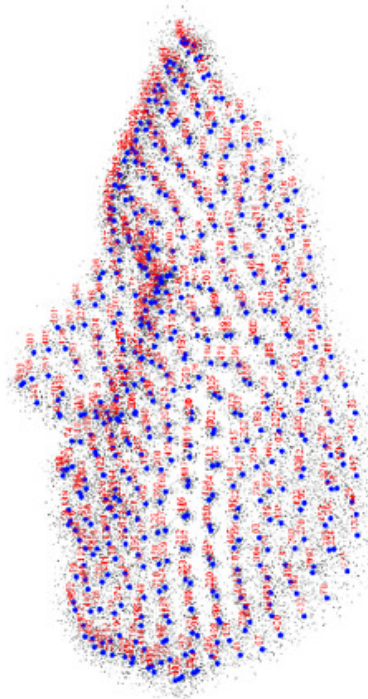

A

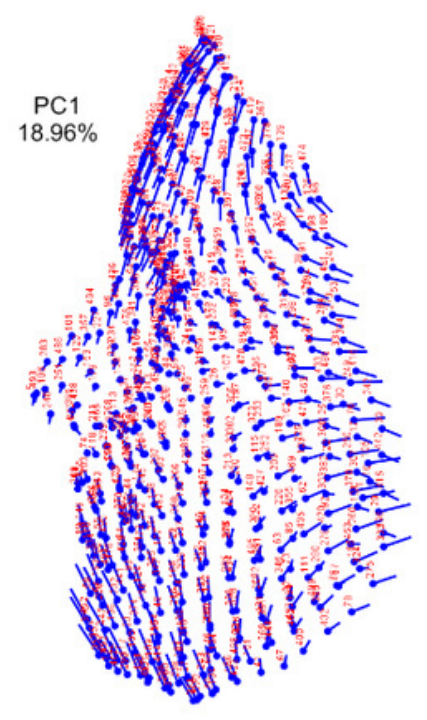

E
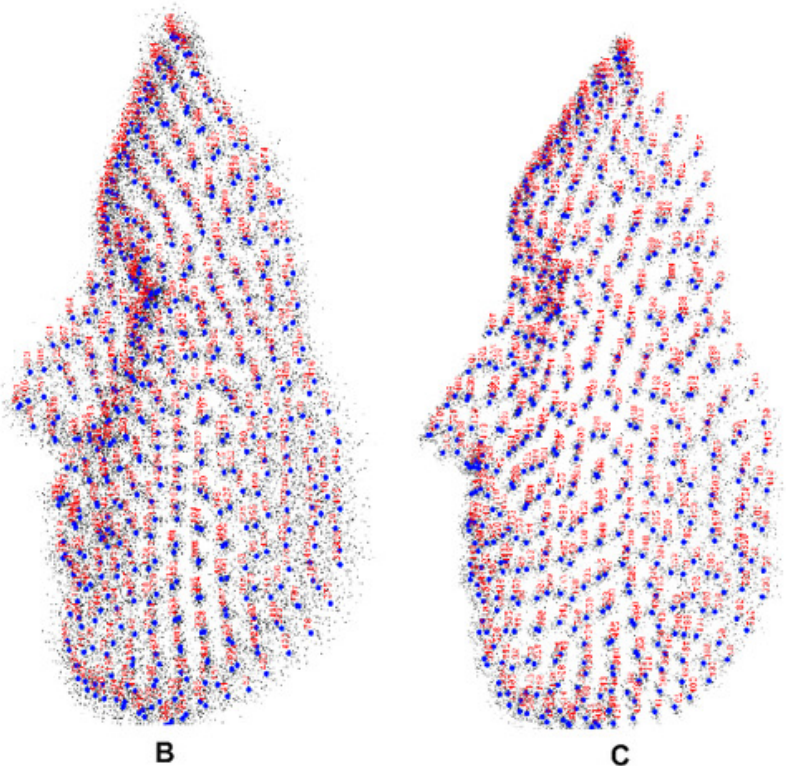

C

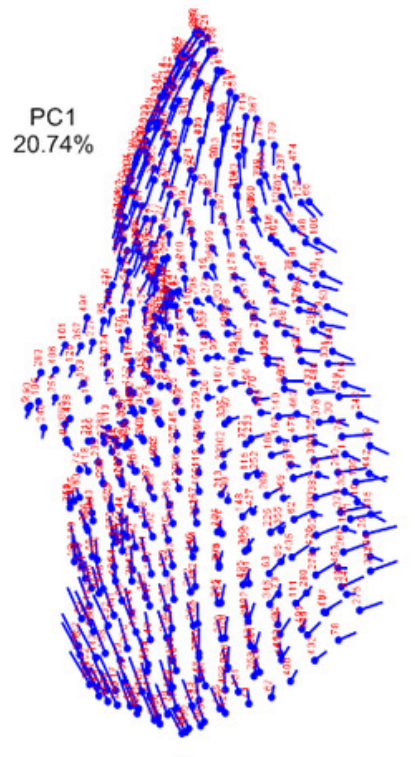

F

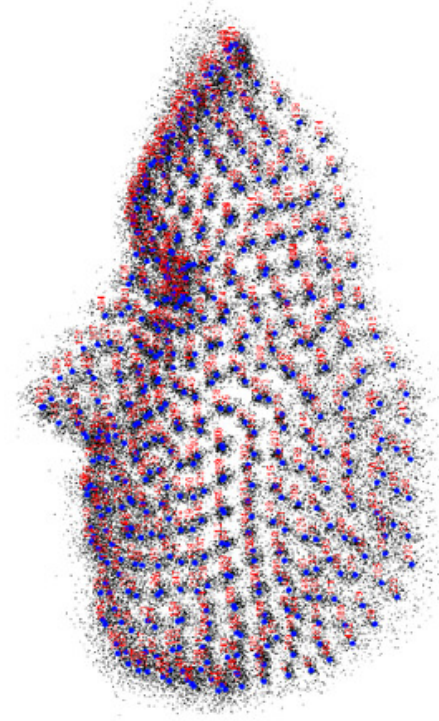

D

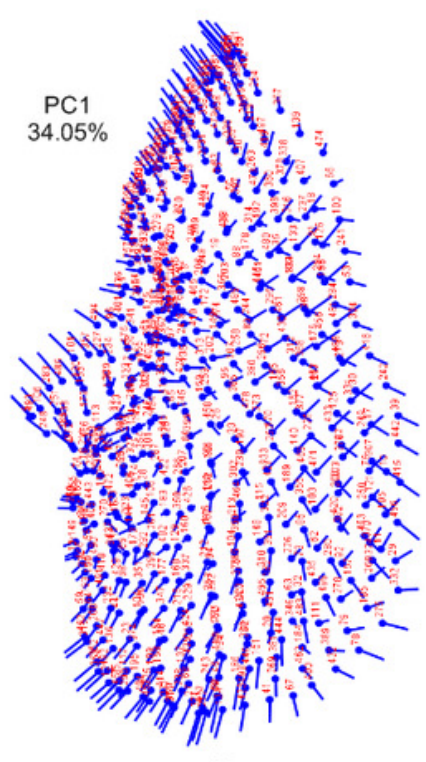

H 


\section{Figure 6}

Principal components, multivariate regression and boxplots for the population groups

(A) Scatterplots of $\mathrm{PC1}$ vs. PC2, which together explain more than $50 \%$ of the variance. Left: manual anatomical landmarks; center: sliding semi-landmarks; right: all landmarks. (B) Allometric regression of population mean shape. (C) Boxplot for centroid size for all populations, showing the size variation after averaging faces within populations.
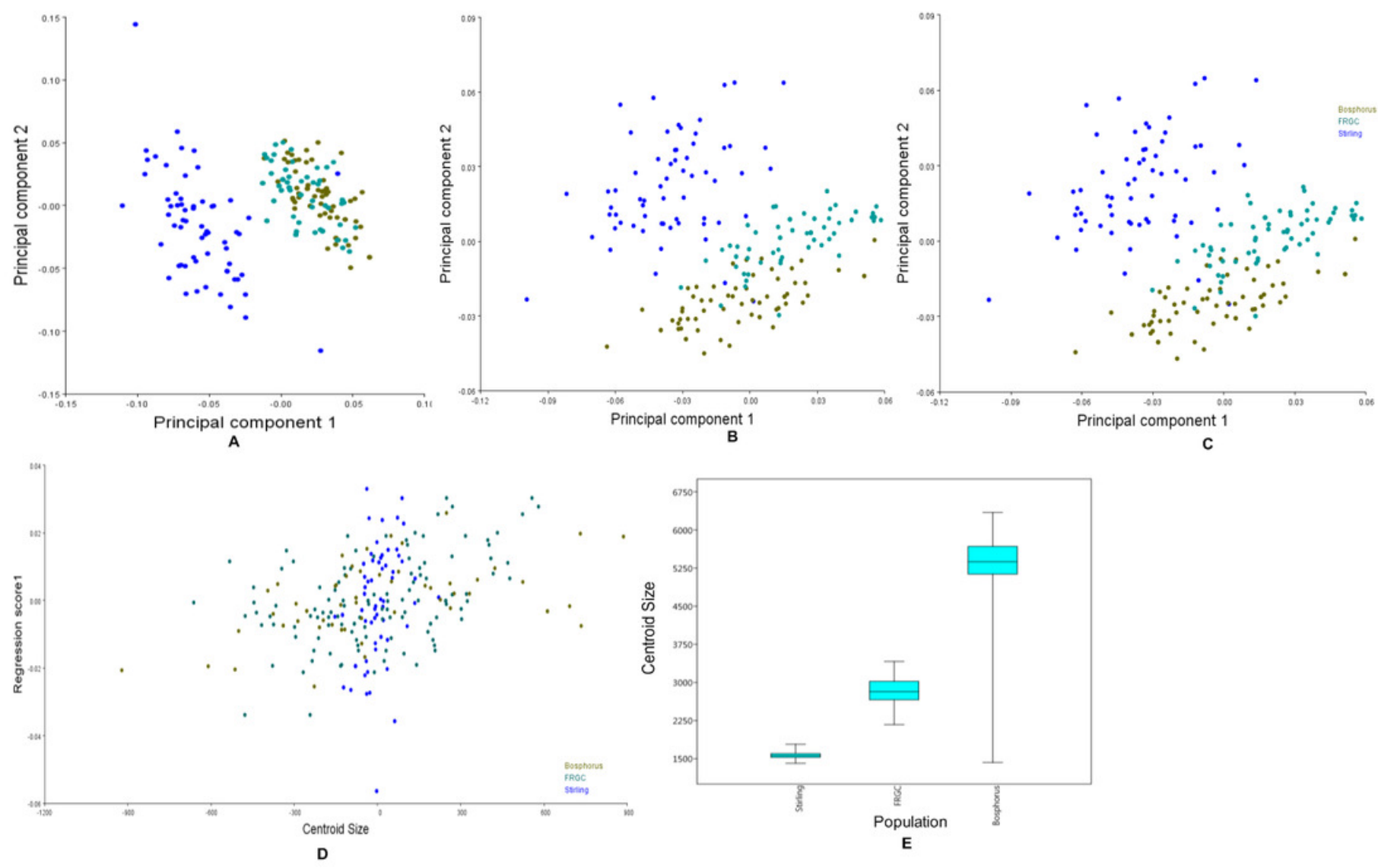


\section{Table 1 (on next page)}

Fixed anatomical landmarks and descriptions 
1 Table 1: Fixed anatomical landmarks and descriptions

\begin{tabular}{|c|c|c|c|}
\hline No & Fixed Landmarks & $\begin{array}{l}\text { 3D } \\
\text { Notation }\end{array}$ & Description \\
\hline 1 & Endocanthion left & enl & $\begin{array}{l}\text { Left most medial point of the palpebral fissure, at } \\
\text { the inner commissure of the eye }\end{array}$ \\
\hline 2 & Exocanthion left & exl & $\begin{array}{l}\text { Left most lateral point of the palpebral fissure, at } \\
\text { the outer commissure of the eye }\end{array}$ \\
\hline 3 & Exocanthion right & exr & $\begin{array}{l}\text { Right most lateral point of the palpebral fissure, at } \\
\text { the outer commissure of the eye }\end{array}$ \\
\hline 4 & Endocanthion right & enr & $\begin{array}{l}\text { Right most medial point of the palpebral fissure, at } \\
\text { the inner commissure of the eye }\end{array}$ \\
\hline 5 & Sellion & se & Deepest midline point of the nasofronal angle \\
\hline 6 & Pronasale & $\mathrm{pr}$ & $\begin{array}{l}\text { The most anteriorly protruded point of the apex } \\
\text { nasi }\end{array}$ \\
\hline 7 & subnasale & su & $\begin{array}{l}\text { Median point at the junction between the lower } \\
\text { border of the nasal septum and the philtrum area }\end{array}$ \\
\hline 8 & Alare left & all & Left most lateral point on the nasal ala \\
\hline 9 & Alare right & alr & Right most lateral point on the nasal ala \\
\hline 10 & Cheilion left & chl & $\begin{array}{l}\text { Left outer corners of the mouth where the outer } \\
\text { edges of the upper and lower vermilions meet }\end{array}$ \\
\hline 11 & Cheilion right & chr & $\begin{array}{l}\text { Right outer corners of the mouth where the outer } \\
\text { edges of the upper and lower vermilions meet }\end{array}$ \\
\hline 12 & Labiale superius & ls & Midpoint of the vermilion border of the upper lip \\
\hline 13 & Labiale inferius & li & Midpoint of the vermilion border of the lower lip \\
\hline 14 & Gnathion & gn & $\begin{array}{l}\text { Median point halfway between pogonion and } \\
\text { menton }\end{array}$ \\
\hline 15 & Obelion left & obl & $\begin{array}{l}\text { Left median point where the sagittal suture } \\
\text { intersects with a transverse line connecting parietal } \\
\text { foramina }\end{array}$ \\
\hline 16 & Obelion right & obr & $\begin{array}{l}\text { Right median point where the sagittal suture } \\
\text { intersects with a transverse line connecting parietal } \\
\text { foramina }\end{array}$ \\
\hline
\end{tabular}




\section{Table 2 (on next page)}

Procrustes ANOVA for facial shape with digitization errors. Top: fixed anatomical landmarks (FAL) only; centre: sliding semi-landmarks (SSL); bottom: combined landmarks (CL).

SS: sum of squares; \%Var: percentage of variance; MS: mean square; DF: degrees of freedom; F: F-statistic; P: P-value (parametric) 
3

4

5

6

7

10

11

12

13

14

Table 2: Procrustes ANOVA for facial shape with digitization errors. Top: fixed anatomical landmarks (FAL) only; centre: sliding semi-landmarks (SSL); bottom: combined landmarks (CL).

\begin{tabular}{|c|c|c|c|c|c|c|}
\hline Effect & Var explained (\%) & SS & MS & DF & $\mathbf{F}$ & $\mathbf{P}$ \\
\hline Population & 25.82 & 0.376064 & 0.004586 & 82 & 42.86 & $<.0001$ \\
\hline Individuals & 69.87 & 1.017737 & 0.000107 & 9512 & 1.05 & 0.2239 \\
\hline Error (FAL) & 4.31 & 0.062844 & 0.000102 & 615 & & \\
\hline Total & 100 & 1.456644 & 0.004795 & 10209 & & \\
\hline Population & 30.41 & 0.215282 & 0.0000744922 & 2890 & 53.56 & $<.0001$ \\
\hline Individuals & 65.88 & 0.466278 & 0.0000013909 & 335240 & 1.15 & $<.0001$ \\
\hline Error (SSL) & 3.71 & 0.026243 & 0.0000012108 & 21675 & & \\
\hline Total & 100 & 0.707804 & 0.0000770939 & 359805 & & \\
\hline Population & 29.48 & 0.218988 & 0.0000733384 & 2986 & 51.15 & $<.0001$ \\
\hline Individuals & 66.85 & 0.496599 & 0.0000014337 & 346376 & 1.18 & $<.0001$ \\
\hline Error (CL) & 3.67 & 0.027269 & 0.0000012176 & 22395 & & \\
\hline Total & 100 & 0.742857 & 0.0000759897 & 371757 & & \\
\hline
\end{tabular}

SS: sum of squares; \%Var: percentage of variance; MS: mean square; DF: degrees of freedom; F: F-statistic; P: P-value (parametric) 


\section{Table 3(on next page)}

MANOVA results in terms of Wilks' Lambda. 
1 Table 3 MANOVA results in terms of Wilks' Lambda

\begin{tabular}{|c|c|c|c|c|c|}
\hline Effect & Wilki & df1 & df2 & $\mathbf{F}$ & $\mathbf{P}$ \\
\hline Population $\mathrm{x}$ CS & 0.0291 & 40 & 456 & 55.43 & $<0.000$ \\
\hline Population & 0.0139 & 42 & 454 & 80.69 & $<0.000$ \\
\hline
\end{tabular}

2 